\title{
A case of pituitary hypophysitis following treatment with ipilimumab
}

F. Iqbal, N. Choudhary, D. Flanagan

Department of Diabetes and Endocrinology, Derriford Hospital, Plymouth Hospitals NHS Trust

\section{INTRODUCTION}

Pituitary hypophysitis is an uncommon but increasingly recognized immune related adverse event seen with ipilimumab. We present an interesting case of ipilimumab induced hypophysitis with partial recovery of hypo pituitarism.

\section{CASE HISTORY}

A 65 year old lady with history of enucleation of left eye for malignant choroidal melanoma was under oncology follow up when a routine surveillance scan showed recurrence in segment six of the liver. A diagnostic laparoscopy showed multi-focal metastatic disease and she was commenced on palliative chemotherapy. Due to disease progression following three doses of dacarbazine and then a combination of a MEK (mitogen activated protein kinase) inhibitor and a protein kinase $C$ inhibitor it was decided to initiate treatment with standard dose $(3 \mathrm{mg} / \mathrm{kg})$ ipilimumab. Two weeks after the second infusion of ipilimumab she developed a moderately severe headache with left eye pressure and difficulty closing left eye. An urgent MRI scan was done which is as follows.
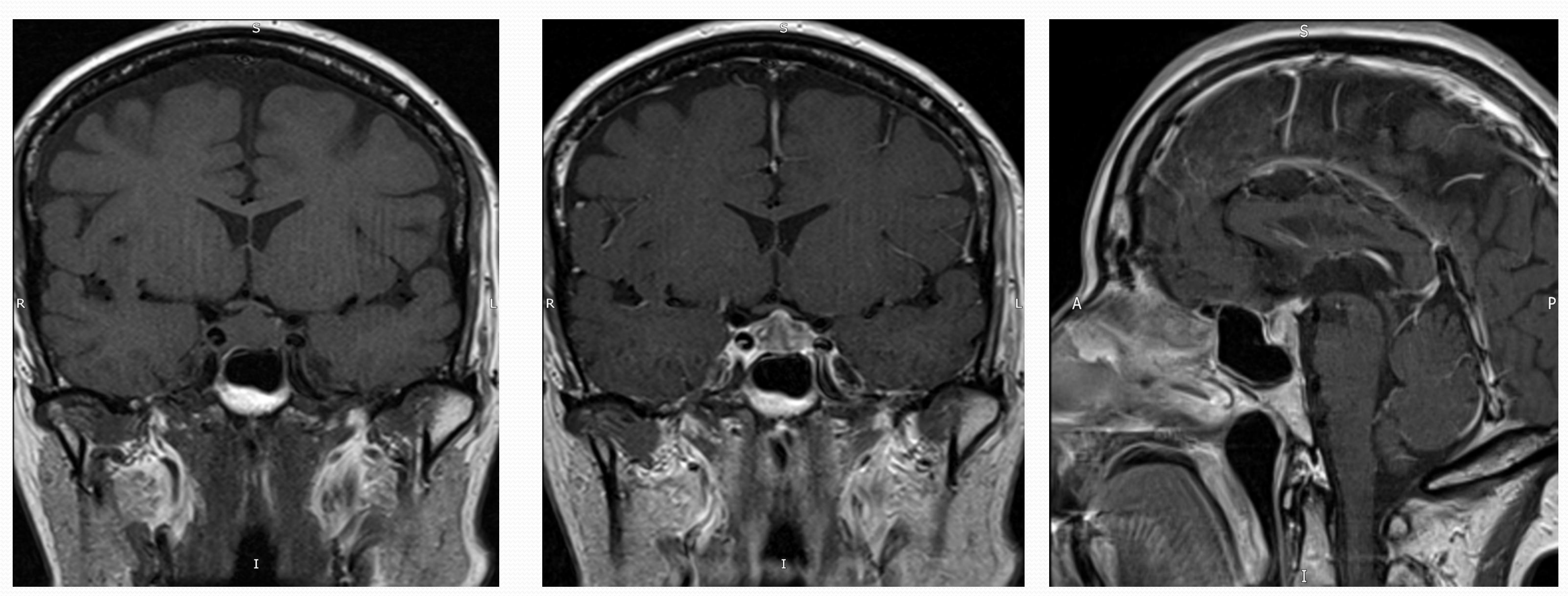

Her pituitary profile as below, confirmed anterior pituitary dysfunction. No cranial nerve palsies were noted on clinical examination and formal visual field testing did not show any defects.

\begin{tabular}{l|l}
\hline Cortisol (nmol/L) & 64 \\
\hline TSH (miu/L) & 0.05 \\
\hline Free T4 (pmol/L) & $7 \cdot 3$ \\
\hline FSH (iu/L) & $3 \cdot 3$ \\
\hline LH (iu/L) & 3.0 \\
\hline Estradiol (pmol/L) & $<44$ \\
\hline Prolactin (miu/L) & 225 \\
\hline IGF-1 (nmol/L) & 30.4
\end{tabular}

She was commenced on dexamthasone $16 \mathrm{mg}$ bd with resolution of her symptoms soon after.. This was gradually tapered and she was transitioned to physiological dose of hydrocortisone by three months. She did not report any symptoms suggestive of diabetes insipidus and a water deprivation test was not performed.
Pituitary testing at six weeks is as below showing obvious improvement of her thyroid and gonadal axis.

\begin{tabular}{|l|l|}
\hline TSH (miu/L) & 1.6 \\
\hline Free T4 (pmol/L) & 16.1 \\
\hline FSH (iu/L) & 25.3 \\
\hline LH (iu/L) & 11.8 \\
\hline
\end{tabular}

Follow up MRI at four months also showed notable improvement as below

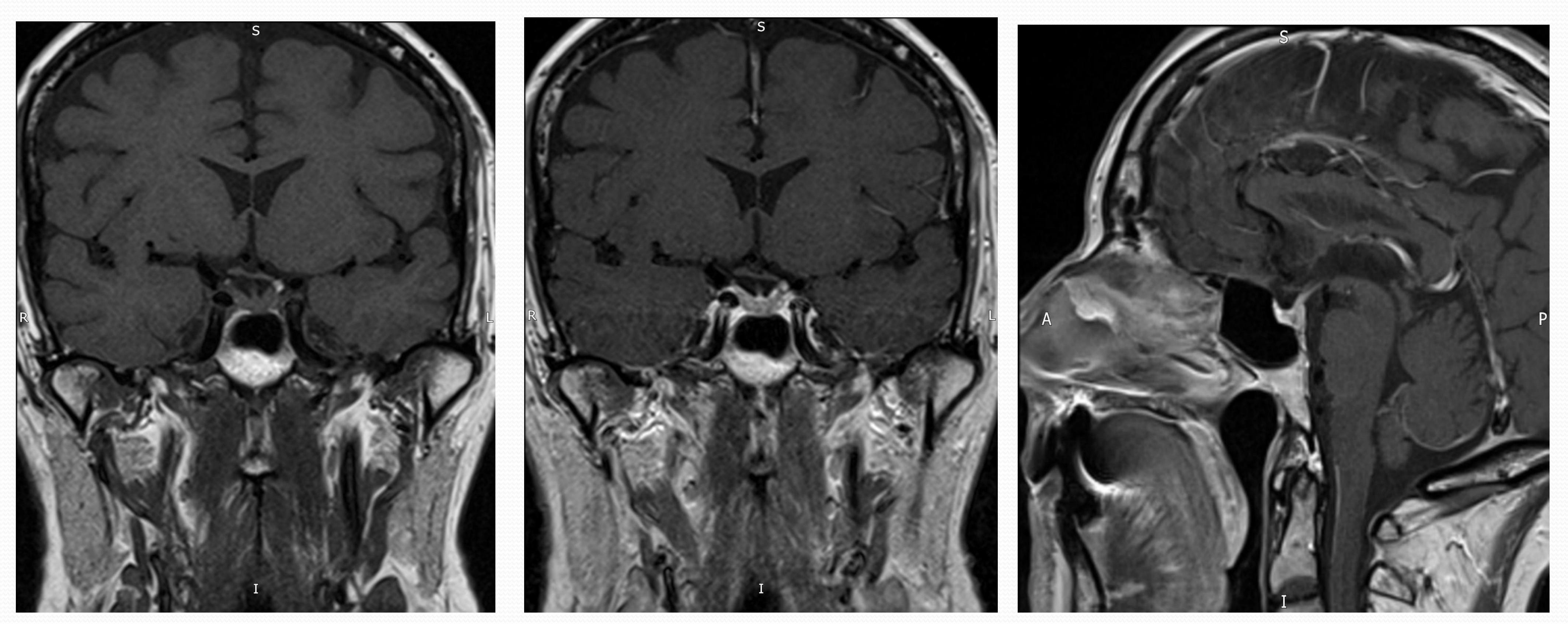

An insulin stress test performed at 6 months showed persistent secondary adrenal insufficiency with a peak cortisol of $307 \mathrm{nmol} / \mathrm{L}$ only. Several months later our patient had an acute admission for pulmonary embolism and progressive hepatic metastatic disease with rapid clinical deterioration and she passed away.

\section{DISCUSSION}

Ipilimumab is a monoclonal antibody which blocks the cytotoxic Tlymphocyte antigen 4 on activated $\mathrm{T}$ cells. In doing so, it prevents $\mathrm{T}$-cell inactivation and augments the anti-tumour response. The most common endocrine immune related adverse event associated with ipilimumab is hypophysitis with a reported incidence ranging from o to $17 \%$. Predisposing factors include male gender and old age. Headache and fatigue are common presenting symptoms while visual impairment is rare. Anterior pituitary dysfunction is the predominant feature with secondary adrenal insufficiency and central hypothyroidism as the most commonly reported abnormalities. Diabetes insipidus has not been reported in this group of hypophysitis. In the majority of cases, hypo pituitarism is persistent. Time to development of hypophysistis can be fairly variable, ranging between 2-12 weeks. MRI features include pituitary enlargement, post contrast homogenous or heterogenous enhancement and stalk thickening. Optic chiasm involvement is rare. Management consists of prompt recognition and physiological dose hormone replacement as needed, with close follow up and periodic imaging. Most retrospective studies note that high dose steroids should be reserved for patients with severe headache, symptomatic adrenal insufficiency with severe hyponatremia or substantial pituitary enlargement on imaging.

\section{CONCLUSION}

With the increasing use of immune checkpoint inhibitors in treatment of advanced cancers, clinicians need to have a heightened awareness of this immune mediated endocrine complication.

\section{REFERENCES}

Corsello, Barnabei, Marchetti. Endocrine Side Effects Induced by Immune Check point Inhibitors. Journal of Clinical Endocrinology and Metabolism. March 2013

Ryder M, Callahan M, Postow MA. Endocrine related adverse events following ipilimumab in patients with advanced melanoma: a comprehensive retrospective review from a single institution

Endocrine Relat Cancer. March 2014

Faje AT, Sullivan R, Lawrence D. Ipilimumab Induced Hypophysitis: A Detailed Longitudinal Analysis in a Large Cohort of Patients with Metastatic Melanoma. Journal of Clinical Endocrinology and Metabolism. July 2014 